\title{
Effect of olfactory manganese dose on motor coordination in iron-deficient rats
}

\begin{abstract}
While exposures to excessive amount of metals increase brain damage, our recent study demonstrated that manganese $(\mathrm{Mn})$ exposure corrected neurobehavioral problems resulting from iron deficiency in young rats. To further characterize the dose-dependent effect of intranasal manganese on motor coordination under iron deficiency, weanling rats were fed iron-deficient $(5 \mathrm{mg}$ iron $/ \mathrm{kg}$ diet $)$ or iron-adequate control diet $(50 \mathrm{mg} / \mathrm{kg})$ for 5 weeks and manganese chloride $(\mathrm{MnCl} 2)$ solution was intranasal instilled through the right nostril twice a week for 3 weeks. Iron-deficient rats displayed lower blood hematocrit than controls, reflecting an iron-deficient anemic condition. Mn instillation did not alter hematocrit but modestly decreased body weight. In the rotarod test, Mn-instilled rats decreased motor coordination compared with water-instilled control rats $(17 \%$ decrease in the time of the first drop; $\mathrm{P}=0.042$ ). Iron-deficiency also decreases rotarod performance. However, upon $\mathrm{Mn}$ instillation, iron-deficient rats stayed longer on the bar by $30 \%$ than controls $(\mathrm{P}=0.006)$. Interestingly, the improvement in motor coordination was associated with manganese dose. Since both iron and Mn support tyrosine hydroxylase (TH), a critical enzyme in dopamine production, we tested if TH expression was modified by Mn instillation under iron deficiency. TH levels in the striatum were increased in iron deficiency and decreased upon $\mathrm{Mn}$ instillation, indicating that TH is unrelated with improved motor coordination in response to olfactory Mn under iron deficiency. Taken together, these results demonstrate that either olfactory $\mathrm{Mn}$ exposure or iron deficiency decreases motor function but a combination of the two restores poor motor performance by $\mathrm{Mn}$ dose-dependent manner.
\end{abstract}

Keywords: striatum, motor coordination, iron deficiency, olfactory manganese, tyrosine hydroxylase, rotarod
Volume I Issue I - 2014

\section{Siripan Phattanarudee, Murui Han, Jonghan \\ Kim}

Department of Pharmaceutical Sciences, Northeastern University, USA

Correspondence: Jonghan Kim, Northeastern University, Department of Pharmaceutical Sciences, 360 Huntington Avenue, Boston, MA 02115 USA; Tel 6173733214, Fax 6I73738886,Email j.kim@neu.edu

Received: August II, 2014 | Published: November 26, 2014
Abbreviations: DAT, dopamine transporter; DMT1, divalent metal transporter $1 ; \mathrm{D}_{1} \mathrm{R}$, dopamine receptor $1 ; \mathrm{D}_{2} \mathrm{R}$, dopamine receptor 2; Fe, iron; ID, iron deficiency; IDA, iron-deficient anemia; Mn, manganese; PND, postnatal day; $\mathrm{TH}$, tyrosine hydroxylase

\section{Introduction}

Iron $(\mathrm{Fe})$ is an essential metal that plays important roles in physiology. Iron is responsible for a vast array of biochemical reactions, including supporting cell division, oxygen transport and mitochondrial function. ${ }^{1,2}$ Iron deficiency is the most prevalent single nutrient deficiency worldwide ${ }^{2,3}$ and results in anemia, retarded growth, fatigue, muscle weakness and shortness of breath., ${ }^{2,45}$ Iron is also required in the brain for neurotransmitter synthesis, neurotransmitter signaling, and myelin synthesis of the oligodendrocytes. ${ }^{6,7}$ Many neurological disorders associated with iron deficiency have been reported. For example, iron deficiency has been associated with poorer fine and gross motor skills, visual-motor integration, language and global IQ, accompanying higher scores in anxiety and depression as well as social and attention problems. ${ }^{8-10}$

Manganese (Mn), another essential trace element, is also needed for many vital functions, including metabolic processes and anti-oxidant capacities. In particular, manganese superoxide dismutase (Mn SOD) catalyzes dismutation of superoxide anion to hydrogen peroxide and molecular oxygen. ${ }^{11}$ Manganese is also involved in bone formation and metabolism of amino acids, cholesterol and carbohydrates. ${ }^{12}$ While Mn deficiency is rarely observed in humans, $\mathrm{Mn}$ exposure is significantly associated with neurobehavioral deficits. Manganese neurotoxicity resembles Parkinson's disease and this has been well-documented in people drinking contaminated water, workers employed in mining and $\mathrm{Mn}$ ore processing and agricultural workers exposed to $\mathrm{Mn}$ containing pesticide. ${ }^{13}$ The use of methylcyclopentadienyl manganese tricarbonyl (MMT), an octane enhancer in gasoline, continues to be a huge concern about potential neurological damage due to inhalable $\mathrm{Mn}$ particles after combustion. ${ }^{14} \mathrm{Mn}$ intoxication has also been observed in children under long-term parenteral nutrition and patients with chronic liver diseases. ${ }^{15}$ Advances in molecular physiology and toxicology have revealed that Mn neurotoxicity is, at least, mediated by dopaminergic dysfunction. ${ }^{16-19}$ The dopaminergic neurotransmission is also impaired by iron deficiency. There is an accumulating body of evidence indicating that iron deficiency reduces dopamine receptor 1 $\left(D_{1} R\right)$ and $D_{2} R$ levels. ${ }^{20-23}$. In addition, extracellular dopamine level is increased in iron-deficient animals, which is related to a reduced uptake by dopamine transporter (DAT), ${ }^{20,24-26}$ and returns to normal levels when brain iron levels are corrected. ${ }^{24,26}$ With respect to Mn, a significant increase in $\mathrm{D}_{1} \mathrm{R}$ and $\mathrm{D}_{2} \mathrm{R}$ protein levels was reported in rats exposed to Mn over postnatal day (PND), ${ }^{1-21}$ particularly in prefrontal cortex and nucleus accumbens. ${ }^{18}$ Treating adult rats and mice with $\mathrm{Mn}$ also increases the number of $\mathrm{D}_{2}$ binding sites in the dorsal striatum. ${ }^{27}$ In addition, rats exposed to $\mathrm{Mn}$ during $\mathrm{PND}^{1-21}$ demonstrated a significant reduction in DAT levels that can persist into adulthood. ${ }^{19,28}$

An accumulating body of evidence has indicated that Mn absorption is enhanced in iron-deficient anemia (IDA) due to iron-responsive upregulation of divalent metal transporter-1 (DMT1) which is expressed 
in intestinal and olfactory epithelium. ${ }^{17,29-31}$ Our previous study demonstrated that iron deficiency impairs motor coordination, which was corrected by olfactory Mn exposure, suggesting a possibility that $\mathrm{Mn}$ could modulate or compensate for some detrimental effects resulting from iron-deficient state. ${ }^{17}$ However, the dose-dependent effect of $\mathrm{Mn}$ to correct the neurobehavioral problem in anemic status is not yet determined. The objective of this study is to determine the dose-dependent effect of olfactory Mn on motor dysfunction found in iron-deficient anemic rats.

\section{Materials and methods}

\section{Animals and diets}

Animal protocols were approved by the Division of Laboratory Animal Medicine (DLAM) and the Northeastern UniversityInstitutional Animal Care and Use Committee (NU-IACUC). Weanling Sprague-Dawley rats were purchased from Charles River Laboratories (Wilmington, MA) and assigned into two groups according to the ranked body weight. Male rats were used because estrogen affects iron metabolism. ${ }^{32,33}$ Rats were maintained on a 12:12-h light-dark cycle and given water ad libitum provided by DLAM. Rats were fed either iron-deficient diet containing 5mg iron/ kg diet (TD.99397, Harlan Teklad, and Madison, WI) or iron-adequate control diet containing $50 \mathrm{mg}$ iron/ $\mathrm{kg}$ diet (TD.07800) for 5 weeks to establish iron-deficient and control cohorts, respectively.

\section{Intranasal instillation of manganese}

In order to establish the dose-response relationship of olfactory manganese in iron deficiency, rats fed iron-deficient diet were intranasally instilled with $\mathrm{MnCl} 2(0 \mathrm{mg} / \mathrm{kg}$ as distilled water, 1,3 , or $10 \mathrm{mg} / \mathrm{kg}$ body weight; Sigma-Aldrich) at the age of 4-week-old. To determine the interaction effect between olfactory $\mathrm{Mn}$ and iron deficiency (i.e., differential effect of intranasal Mn between irondeficient and control rats), rats fed control diet were also intranasally instilled with either distilled water $(0 \mathrm{mg} / \mathrm{kg})$ or $\mathrm{MnCl} 210 \mathrm{mg} / \mathrm{kg}$. Intranasal Mn was administered into the right nostril with thin gel loading tips $(0.04 \mathrm{~mL} / \mathrm{kg})$ under isoflurane anesthesia twice a week for 3 weeks.

\section{Rotarod test}

One week after the last dose of intranasal instillation, rats (7.5-week-old) were placed on a standard accelerating rotarod device (Harvard Apparatus). Following 3-day training sessions at fixed speeds $(4,7$ and 10rpm on Day 1; 7, 10 and 13rpm on Day 2; 10, 13 and 16rpm on Day 3), rats were tested twice on the rotarod with accelerating speeds from 4 to $40 \mathrm{rpm}$ over 5 -min (maximum time on bar) or until the rats fell off from the device. ${ }^{17}$ The rotarod device was cleaned between trials using Quatricide TB (Pharmaceutical Research Laboratories Inc., Waterbury, CT). Time of the first drop was recorded and the better score of the two trials was used for analysis. ${ }^{17}$

\section{Tissue collection}

Rats at the age of 8-week-old were euthanized by isoflurane overdose, followed by exsanguinations and collection of brain. Hematocrit values were determined after blood centrifugation. All tissues were flash-frozen in liquid nitrogen and stored at $-80^{\circ} \mathrm{C}$ until analysis.

\section{Western blot analysis}

The right striatum harvested by brain micro dissection was homogenized, electrophoresed on $10 \%$ SDS-polyacrylamide gels $(40 \mu \mathrm{g}$ proteins) and transferred to nitrocellulose membranes. After blocking with $5 \%$ non-fat milk, the membrane was incubated in mouse antibody against tyrosine hydroxylase (1:500; Santa Cruz, Dallas, TX). As a loading control, the immunoblot was incubated with mouse anti-actin (1:5,000, MP Biomedicals, Solon, OH). The blots were incubated with sheep anti-mouse antibody conjugated with HRP $(1: 1,000$, GE Healthcare, Piscataway, NJ), subjected to chemiluminescence (ECL West Dura, Thermo Scientific) and scanned using Chemidoc System (ChemiDoc XRS, Bio-Rad, Hercules, CA). Relative intensities of protein bands normalized to actin were determined using Image Lab (version 4.1; Bio-Rad).

\section{Statistical analysis}

Values reported were expressed as means \pm SEM. A two-way ANOVA was performed using Sigma Plot (version 12.3; Systat Software Inc., San Jose, CA) to determine individual main effects (Mn exposure and iron deficiency) and an interaction effect between iron deficiency and olfactory manganese exposure. A one-way ANOVA was performed to determine dose-dependent Mn effect in iron deficiency, followed by the Holm-Sidakpost-hoc analysis for multiple comparisons. Differences were considered significant at $\mathrm{P}<$ 0.05 .

\section{Results and discussion}

Intranasal manganese instillation retards growth in iron-adequate status but not in iron deficiency. Weanling rats fed either iron-adequate control diet $(50 \mathrm{mg} / \mathrm{kg}$ diet $)$ or iron-deficient diet $(5 \mathrm{mg} / \mathrm{kg})$ received intranasal instillation of manganese chloride $\left(\mathrm{MnCl}_{2}\right)$ or distilled water (as vehicle control) for 3 weeks (Figure 1) and body weight changes were measured to characterize the influence of olfactory manganese and iron deficiency on growth (Figure 2). All rats studied gained body weight. There was a significant difference in body weight between control and iron-deficient rats $(\mathrm{P}=0.026, \mathrm{n}=7-9$ per group $)$ at the age of 6-week-old, but this difference did not persist in later ages (Figure 2A). In contrast, manganese instillation modestly decreased body weight at 6 -week-old age $(4-11 \%$ decrease; $\mathrm{P}=0.044, \mathrm{n}=8-9$ per group) and this difference continued up to 8 -week-old. These results indicate a negative influence of $\mathrm{Mn}$ exposure and iron deficiency on growth in young rats.

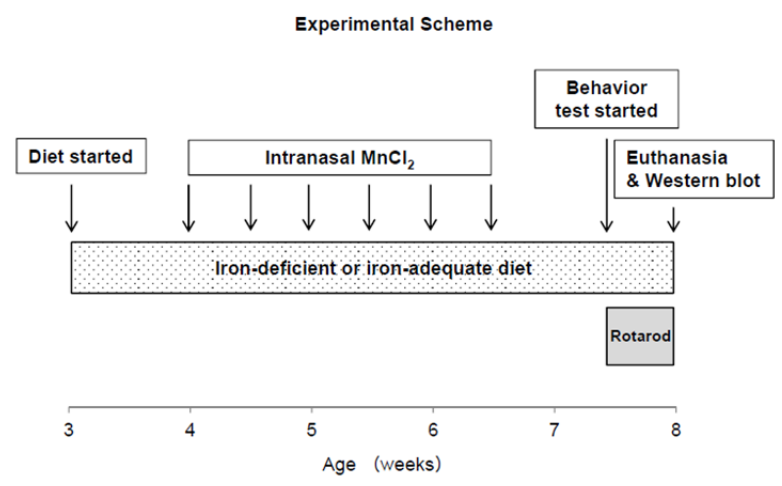

Figure I Experimental scheme of the study.

Weanling male Sprague-Dawley rats were fed either iron-deficient diet or control diet for 5 weeks and intranasal instilled with $\mathrm{MnCl} 2(0, \mathrm{I}, 3$ and I0mg/ $\mathrm{kg}$ ). After rotarod test, rats were euthanized and brain tissues were collected for western blot analysis 

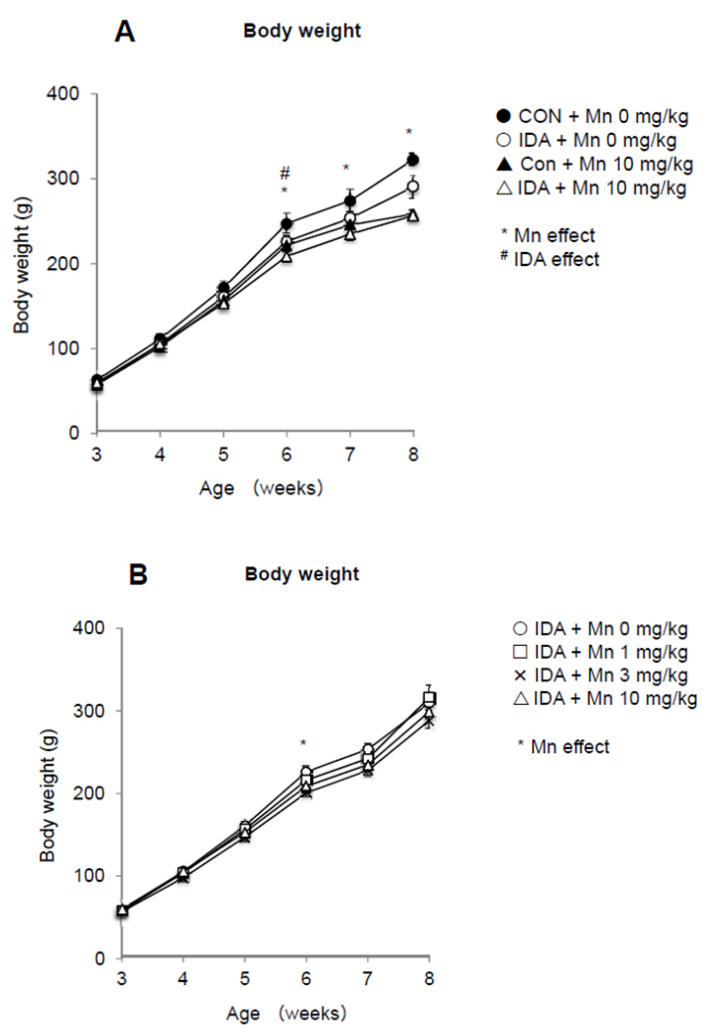

Figure 2 Body weight change in iron-deficient and control rats intranasalinstilled with manganese chloride.

Weanling male Sprague-Dawley rats were fed either iron-deficient diet or control diet for 5 weeks and intranasal instilled with $\mathrm{MnCl} 2(0$ and $10 \mathrm{mg} / \mathrm{kg}$ into control rats; $0,1,3$ and $10 \mathrm{mg} / \mathrm{kg}$ into iron-deficient rats). Body weight was measured weekly. Data were presented as mean \pm SEM ( $n=7-9$ per group) and analyzed using ANOVA.*P $<0.05$ by $\mathrm{Mn}$ effect; $\# \mathrm{P}<0.05$ by iron-deficient anemia (IDA) effect.

We also tested if olfactory Mn could alter the body weight gain under iron deficiency (Figure 2B). When the rats were 6-week-old, $\mathrm{Mn}$ instillation decreased body weight compared with non-exposed iron-deficient rats (Figure 2B). Unlike iron-adequate rats (Figure 2A), however, $\mathrm{MnCl}_{2}$ instillation did not show a significant decrease in body weight in later ages upon iron deficiency (Figure 2B). It might be speculated that Mn could serve as iron for growth when the body is deficient in iron. For example, under iron deficiency some ironrequiring components (e.g., tyrosine hydroxylase) could incorporate and utilize excessive $\mathrm{Mn}$, which otherwise is toxic and inhibits cellular metabolism. ${ }^{17}$ It is also equally possible that a greater availability of iron transport molecules (e.g., transferring) under iron deficiency ${ }^{34}$ could capture manganese excess and store/transport as inactive and less toxic form. Biochemical studies designed for iron-manganese interaction would help to identify these potential mechanisms.

Intranasal manganese does not alter hematocrit. Since iron is an essential component for red blood cell production, we tested if

manganese exposure could directly or indirectly affect hematocrit values rats in normal and iron-deficiency state (Figure 3). As expected, iron-deficient rats demonstrated significantly decreased hematocrit (Figure $3 \mathrm{~A})(\mathrm{P}=0.002$ as overall IDA effect; $\mathrm{n}=4$ per group), while $\mathrm{Mn}$ instillation did not modify hematocrit regardless of iron status. Also, there was no dose effect of $\mathrm{Mn}$ in iron deficiency (Figure 3B) $(\mathrm{P}=0.599$ as overall Mn effect). Taken together, olfactory Mn exposures impair normal growth, especially when the body iron is sufficient (normal), without altering hematocrit.

Olfactory manganese reverses anemia-induced motor dysfunction by a dose-dependent manner. In order to test if olfactory Mn exposure improves attenuated motor function caused by dietary iron deficiency, we carried out motor coordination test using a standard rotarod device after intranasal instillation of $\mathrm{Mn}$ to rats fed control and iron-deficient diets with different doses of $\mathrm{MnCl}_{2}(0,1,3$ and $10 \mathrm{mg} / \mathrm{kg})$. There were several important findings (Figure 4). First, Mn-instilled rats fed ironadequate control diet diminished motor skill by $17 \%$, as evidenced by the shortened time of the first drop from the bar compared with water-instilled rats (Figure $4 \mathrm{~A})(146 \pm 9 \mathrm{sec}$ vs $176 \pm 11 \mathrm{sec}, \mathrm{n}=8-12$ per group; $\mathrm{P}=0.042$ ). Second, iron-deficient rats instilled with $\mathrm{Mn}$ stayed on the bar longer compared with $\mathrm{Mn}$-instilled iron-adequate rats $(30 \%$ increase; $189 \pm 7$ vs $146 \pm 9, \mathrm{n}=7-12$ per group; $\mathrm{P}=0.006)$. This indicates an interaction effect between $\mathrm{Mn}$ instillation and iron status (Figure 3A) (IDA x Mn interaction analyzed by two-ANOVA; $\mathrm{P}=0.008$ ) and further suggests that olfactory $\mathrm{Mn}$ administration rescues iron deficiency-associated motor dysfunction, consistent with our previous findings. ${ }^{17}$ Third, iron-deficient rats exposed to $\mathrm{MnCl}_{2}$ $1 \mathrm{mg} / \mathrm{kg}(152 \mathrm{sec})$ or $3 \mathrm{mg} / \mathrm{kg}(161 \mathrm{sec})$ had similar motor impairment to water-instilled iron-deficient rats $(160 \mathrm{sec})$, whereas only $\mathrm{MnCl}_{2} 10 \mathrm{mg}$ instillation $(189 \mathrm{sec})$ showed a significant increase in motor function by $20 \%$ compared with $\mathrm{MnCl}_{2} 1 \mathrm{mg} / \mathrm{kg}(\mathrm{n}=7-11$ per group; $\mathrm{P}=0.031)$ and exhibited a higher trend (16\% increase) compared with waterinstilled IDA rats $(\mathrm{P}=0.102)$. Combined, these results indicate that intranasal manganese restores motor dysfunction caused by systemic iron deficiency anemia by manganese dose-dependent manner.
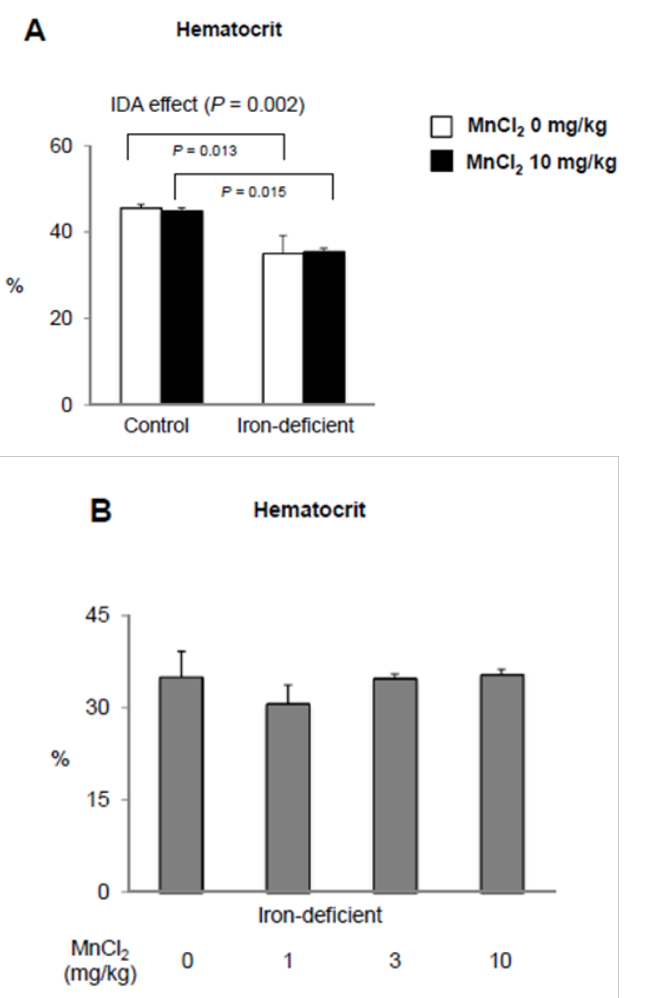

Figure 3 Hematocrit values in iron-deficient and control rats intranasalinstilled with manganese chloride.

Following the rotarod test, rats described in Figure 2 were euthanized and hematocrit values were determined at the age of 8-week-old. Data were presented as mean \pm SEM ( $n=4$ per group) and analyzed using ANOVA. 
Both iron deficiency and manganese overload are associated with motor skill deficits. For instance, in rats, iron-deficiency anemia reduced physical endurance capacity and voluntary use of an activity wheel. ${ }^{35}$ Glover and Jacobs ${ }^{36}$ also reported the reduced spontaneous movements in a 24-hr period with iron-deficiency anemia (mild or severe anemia). Similarly, we have recently reported that iron-deficient rats fell sooner from the bar of a rotarod device with decreased speed. ${ }^{17,37}$ In addition, we have shown that intranasal $\mathrm{Mn}$ at the highest dose $\left(\mathrm{MnCl}_{2} 10 \mathrm{mg} / \mathrm{kg}\right)$ impairs motor function in rats fed normal diet. ${ }^{17}$ We observed the same pattern in the present study and the results are also in accordance with those reported by Cordova et al. ${ }^{38}$ who found the impaired motor coordination in the $3^{\text {rd }}, 4^{\text {th }}$ and $5^{\text {th }}$ week of life after Mn exposures $(10$ and $20 \mathrm{mg} /$ $\mathrm{kg}$ ) during PND 8-12. Also, Vezeret et al. ${ }^{39}$ demonstrated that rats exposed to 15 and $59 \mathrm{mg} / \mathrm{kg} \mathrm{MnCl}_{2}$ via gavage for 10 weeks showed hypo activity, decreased memory performance and diminished sensori motor reaction. However, our data show that motor coordination is not impaired when these two negative influences (i.e., iron deficiency and manganese loading) were applied together, which is consistent with our previous finding. ${ }^{17}$ Our results support an idea that Mn exposure could be beneficial for recovering impaired motor coordination found in the iron-deficient state and further suggest that brain dopaminergic neurotransmission could be involved in the modulation of motor performance. ${ }^{17}$

\section{A Time of First Drop}

IDA $x$ Mn interaction $(P=0.008)$

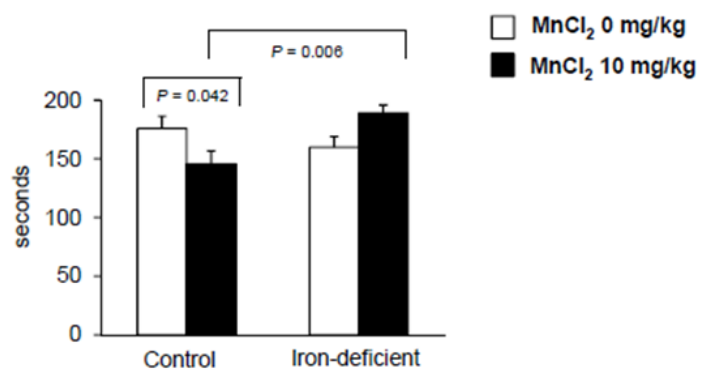

B Time of First Drop

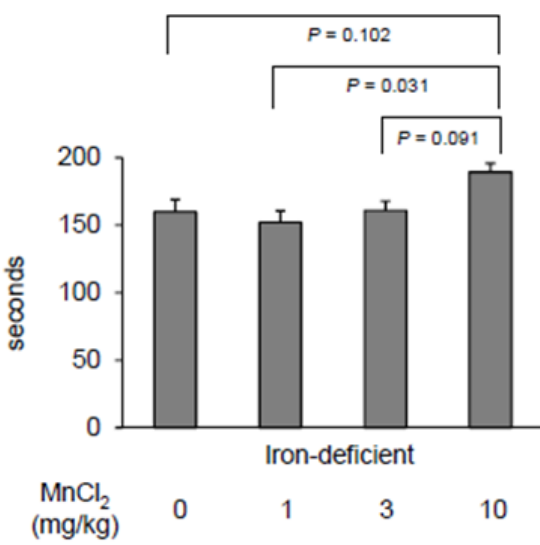

Figure 4 Effect of intranasal manganese instillation and iron deficiency on motor coordination.

Rats intranasal instilled with $\mathrm{MnCl} 2$ or distilled water for 3 weeks were tested on the rotarod device to record the time to falling-off. Data were presented as mean $\pm \operatorname{SEM}(n=7-12$ per group) and analyzed using two-way.

Figure 4A One-way. Figure 4B ANOVA.
Tyrosine hydroxylase is altered upon olfactory Mn exposures and iron deficiency. Although motor coordination is controlled by several neurotransmission pathways, the dopaminergic system in the nigrostriatal pathway has been shown to play a predominant role. ${ }^{40} \mathrm{We}$ have demonstrated that amphetamine-evoked extracellular dopamine levels in the striatum were correlated with motor coordination data upon $\mathrm{Mn}$ exposure in iron deficiency. ${ }^{17}$ However, dopamine-related synaptic proteins, including dopamine transporter (DAT), dopamine receptor $1\left(D_{1} R\right)$ and $D_{2} R$, were not associated with Mn effect on iron deficiency. ${ }^{17}$ Therefore, it was hypothesized that the reversing effect of intranasal Mn instillation on motor dysfunction in iron deficiency could result from altered activity of intracellular proteins related to dopamine homeostasis. ${ }^{17}$ Since tyrosine hydroxylase (TH) is the critical enzyme for dopamine synthesis and since both Fe and Mn can support TH activity, ${ }^{41}$ we characterized the interaction effect between olfactory Mn exposure and iron deficiency on the expression of $\mathrm{TH}$ in the striatum.

We found that both iron and manganese influenced the levels of striatal TH (Figure 5). First, olfactory Mn instillation significantly down-regulated $\mathrm{TH}$ by $78 \%$ in iron-adequate rats (Figure $5 \mathrm{~A})(\mathrm{P}<$ $0.001)$ and by $70 \%$ in iron-deficient rats $(\mathrm{P}<0.001 ; \mathrm{n}=4$ per group $)$, which is in agreement with Zhang et al., ${ }^{42}$ who showed that chronic manganese exposure $(0.1-1 \mu \mathrm{M}$ for $24 \mathrm{~h})$ induced a dose-dependent decrease in TH activity of dopaminergic neuronal cell line. Sriram et al. ${ }^{43}$ also reported that rats exposed to aerosolized welding fumes (manual metal arc-hard surfacing; containing high $\mathrm{Mn}$ ) of $0.5 \mathrm{mg} / \mathrm{rat}$ for 7 weeks caused loss of TH protein in the striatum and midbrain. In addition, a sustained loss was observed in the midbrain 105 days after cessation of Mn exposure..$^{43}$ Second, iron-deficient rats displayed elevated $\mathrm{TH}$ levels by $50 \%$ increase compared with iron-adequate control rats (Figure 5A) $(\mathrm{P}=0.007)$. Upon $\mathrm{Mn}$ exposure, this pattern still existed; Mn-exposed iron-deficient rats had 105\% greater levels of $\mathrm{TH}$ than Mn-instilled iron-adequate rats (Figure $5 \mathrm{~A} ; \mathrm{n}=4$ per group). Our results are in agreement with findings by Unger et al. ${ }^{44}$ that iron-deficiency beginning on gestational day 15 showed increased $\mathrm{TH}$ levels as well as phosphorylated TH levels in prefrontal cortex and striatum at PND 15. However, since iron is required for TH activity, these results do not explain how and why TH protein levels are elevated upon iron deficiency. One possibility is that iron deficiency could reduce TH "activity", which might subsequently have promoted a compensatory mechanism that up-regulates TH "expression levels". Third, in contrast to motor coordination results (Figure 4A), there was no interaction effect between $\mathrm{Mn}$ and iron deficiency (Figure 5A). Finally, we observed $\mathrm{Mn}$ dose-dependent reduction in striatal $\mathrm{TH}$ levels in iron deficiency (Figure 5B), which cannot explain the dosedependent increase in motor performance under IDA status (Figure 4B). These results suggest that changes in TH expression levels are not associated with a beneficial role of manganese in motor coordination.

While we characterized in the present study olfactory uptake of manganese into the brain through the air-brain barrier after intranasal instillation of the metal, manganese transport across the blood-brain barrier (BBB) deserves discussion. Some evidence indicates that both transferring receptor 1 (TfR1) and the divalent metal transporter 1 (DMT1) contribute to Mn transport across the BBB. ${ }^{45,46}$ Other transporters have been reported to possess an ability to transport Mn. For example, Mn influx across the BBB is partially facilitated by choline transporter. ${ }^{47,48}$ Also, the transient receptor potential melastatin 7 (TRPM7) has been shown to act as a channel for divalent cation trace metals, including $\mathrm{Mn} .{ }^{49}$ More studies are necessary to understand the exact role of these transporters in the transport of $\mathrm{Mn}$ and other metals across the BBB. 


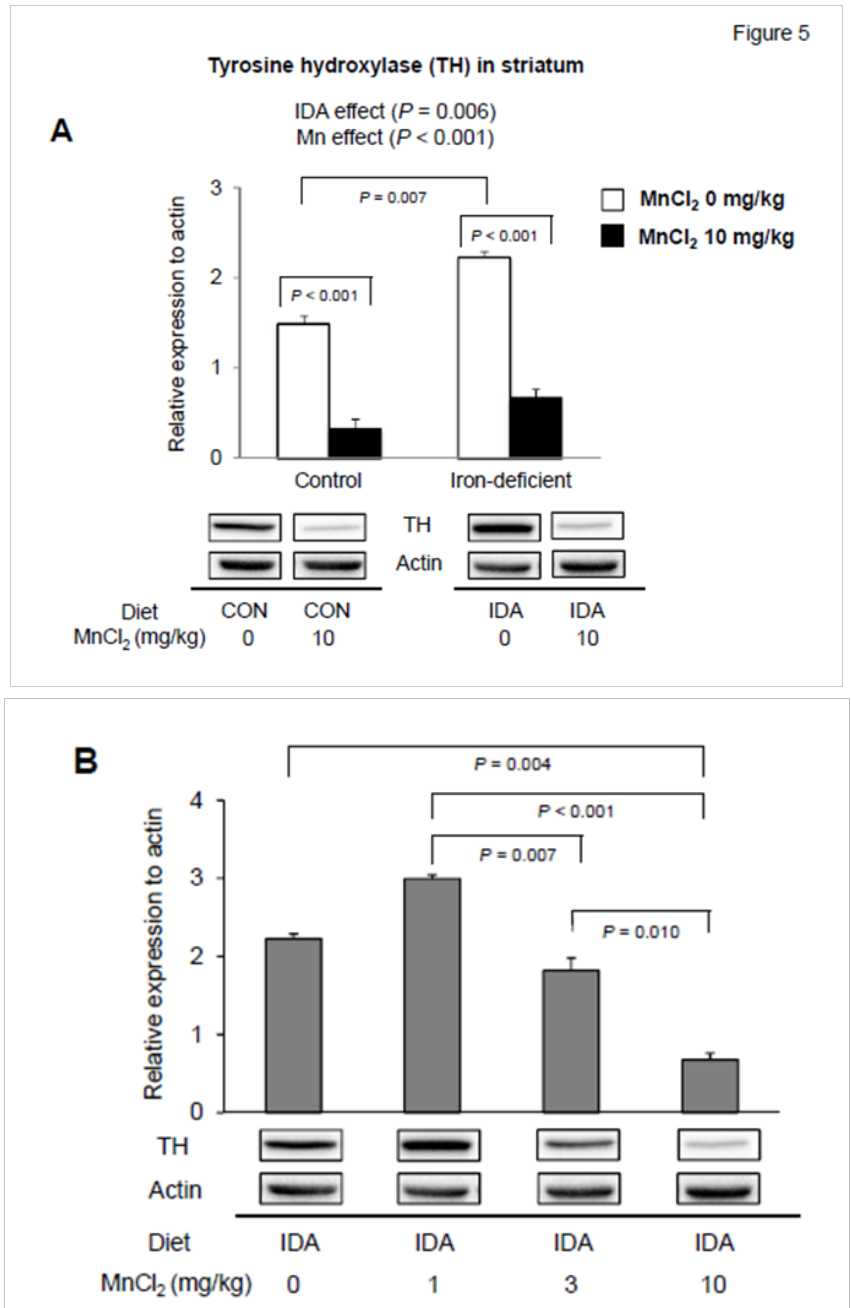

Figure 5 Effect of manganese instillation and iron deficiency on the expression of tyrosine hydroxylase in the striatum. Striatum collected from rats intranasal instilled with $\mathrm{MnCl} 2$ was homogenized to determine the expression levels of tyrosine hydroxylase. Relative intensities of protein bands normalized to actin were determined using Image Lab (version 4.I). Data were presented as mean $\pm \operatorname{SEM}(n=4$ per group) and were analyzed using two-way.

Figure 5A One-way. Figure 5B ANOVA.

Manganese-induced neurotoxicity is most significant when inhaled due to increased olfactory absorption and direct uptake into the brain, providing the relevance to occupational and environmental health and safety. ${ }^{50}$ Our previous study has provided evidence that impaired motor function due to iron deficiency is rescued by olfactory manganese. ${ }^{17}$ Thus, in the present study we characterized manganese dose-response relationship under iron deficiency. To our knowledge, the present study is the first to demonstrate the quantitative effect of olfactory Mn exposure on neurobehavioral problems caused by irondeficient state. Our results demonstrate that either olfactory manganese exposure or iron deficiency reduces motor coordination in rats and that a combination of the two challenges provides a beneficial effect by modulating negative influences of each metal by a manganese-dose dependent manner.

Several possibilities exist to explain dose-dependent effect of olfactory manganese on improved motor skills upon iron deficiency. In the previous and current study, we have excluded the possible influence of TH, DAT, $D_{1} R$ and $D_{2} R$. It remains to be tested if peripheral muscular activity could have been modified by manganese exposure and iron deficiency since motor performance determined by rotarod device would be controlled by both central and peripheral systems. Altered levels of dopamine and expression of dopamine-related proteins in the cerebellum, another important site for maintenance of posture, balance and motor coordination could contribute to olfactory Mn effect on iron deficiency, although the dopaminergic innervations in the cerebellum is rather low. ${ }^{51,52}$ It should also be noted that other potential mechanisms could be involved in behaviors related to iron deficiency/manganese overload and that other neurotransmitters could play significant roles in these behaviors. For example, iron deficiency alters serotoninergic ${ }^{53}$ and GABAergic functions,${ }^{54}$ while cholinergic homeostasis is modified by manganese. ${ }^{55}$ It is also possible that these effects are not due to direct effect by iron and manganese but may be a consequence of other factors such as interactions with other metals, including zinc and copper. Further investigations are warranted to explore these possibilities and to determine the signaling pathways of other neurotransmitters and receptors and their clinical consequences.

\section{Acknowledgements}

None.

\section{Conflict of interest}

The author declares no conflict of interest.

\section{References}

1. Hare D, Ayton S, Bush A, et al. A delicate balance:Iron metabolism and diseases of the brain. Front Aging Neurosci. 2013;5:34

2. Beard JL, Connor JR. Iron status and neural functioning. Annu Rev Nutr. 2003;23:41-58.

3. ACC/SCN. Second report on the world nutrition situation. 1992.

4. Lozoff B, Brittenham GM. Behavioral aspects of iron deficiency. Prog Hematol. 1986;14:23-53.

5. Wood MM, Elwood PC. Symptoms of iron deficiency anaemia. A community survey. Br J Prev Soc Med. 1966;20(3):117-121.

6. Morath DJ, Mayer Proschel M. Iron deficiency during embryogenesis and consequences for oligodendrocyte generation in vivo. Dev Neurosci. 2002;24(2-3):197-207.

7. Beard J. Recent evidence from human and animal studies regarding iron status and infant development. J Nutr. 2007;137(2):524S-530S.

8. Palti H, Meijer A, Adler B. Learning achievement and behavior at school of anemic and non-anemic infants. Early Hum Dev. 1985;10(34):217-223.

9. Lozoff B, Jimenez E, Wolf AW. Long-term developmental outcome of infants with iron deficiency. N Engl J Med. 1991;325(10):687-694.

10. Hurtado EK, Claussen AH, Scott KG. Early childhood anemia and mild or moderate mental retardation. Am J Clin Nutr. 1999;69(1):115-119.

11. Miao L, St Clair DK. Regulation of superoxide dismutase genes: implications in disease. Free Radic Biol Med. 2009;47(4):344-356.

12. Erikson KM, Syversen T, Aschner JL, et al. Interactions between excessive manganese exposures and dietary iron-deficiency in neurodegeneration. Environ Toxicol Pharmacol. 2005;19(3):415-421.

13. Grandjean P, Landrigan PJ. Neurobehavioural effects of developmental toxicity. Lancet Neurol. 2014;13(3):330-338.

14. Davis JM. Methylcyclopentadienyl manganese tricarbonyl: health risk uncertainties and research directions. Environ Health Perspect. 1998;106 Suppl 1:191-201. 
15. Erikson KM, Thompson K, Aschner J, et al. Manganese neurotoxicity: a focus on the neonate. Pharmacol Ther. 2007;113(2):369-377.

16. Benedetto A, Au C, Aschner M. Manganese-induced dopaminergic neurodegeneration: insights into mechanisms and genetics shared with Parkinson's disease. Chem Rev. 2009;109(10):4862-4884.

17. Kim J, Li Y, Buckett PD, et al. Iron-responsive olfactory uptake of manganese improves motor function deficits associated with iron deficiency. PLoS One. 2012;7(3):e33533.

18. Kern $\mathrm{CH}$, Smith DR. Preweaning Mn exposure leads to prolonged astrocyte activation and lasting effects on the dopaminergic system in adult male rats. Synapse. 2011;65(6):532-544.

19. Kern CH, Stanwood GD, Smith DR. Preweaning manganese exposure causes hyperactivity, disinhibition, and spatial learning and memory deficits associated with altered dopamine receptor and transporter levels. Synapse. 2010;64(5):363-378.

20. Erikson KM, Jones BC, Beard JL. Iron deficiency alters dopamine transporter functioning in rat striatum. J Nutr. 2000;130(11):2831-2837.

21. Guilarte TR, Burton NC, McGlothan JL, et al. Impairment of nigrostriatal dopamine neurotransmission by manganese is mediated by pre-synaptic mechanism(s): implications to manganese-induced parkinsonism. J Neurochem. 2008;107(5):1236-1247.

22. Erikson KM, Jones BC, Hess EJ, et al. Iron deficiency decreases dopamine D1 and D2 receptors in rat brain. Pharmacol Biochem Behav. 2001;69(3-4):409-418.

23. Beard JL, Erikson KM, Jones BC. Neurobehavioral analysis of developmental iron deficiency in rats. Behav Brain Res. 2002;134(12):517-524.

24. Chen Q, Beard JL, Jones BC. Abnormal rat brain monoamine metabolism in iron deficiency anemia. Nutr Biochem. 1995;6(9):486-493.

25. Beard JL, Chen Q, Connor J, et al. Altered monamine metabolism in caudate-putamen of iron-deficient rats. Pharmacol Biochem Behav. 1994;48(3):621-624.

26. Nelson C, Erikson K, Pinero DJ, et al. In vivo dopamine metabolism is altered in iron-deficient anemic rats. J Nutr. 1997;127(12):2282-2288.

27. Seth PK, Hong JS, Kilts CD, et al. Alteration of cerebral neurotransmitter receptor function by exposure of rats to manganese. Toxicol Lett. 1981;9(3):247-254.

28. McDougall SA, Reichel CM, Farley CM, et al. Postnatal manganese exposure alters dopamine transporter function in adult rats: Potential impact on nonassociative and associative processes. Neuroscience. 2008;154(2):848-860.

29. Thompson K, Molina RM, Donaghey T, et al. Olfactory uptake of manganese requires DMT1 and is enhanced by anemia. FASEB $J$. 2007;21(1):223-230.

30. Hansen SL, Trakooljul N, Liu HC, et al. Iron transporters are differentially regulated by dietary iron, and modifications are associated with changes in manganese metabolism in young pigs. $J$ Nutr. 2009;139(8):1474-1479.

31. Seo YA, Li Y, Wessling Resnick M. Iron depletion increases manganese uptake and potentiates apoptosis through ER stress. Neurotoxicology. 2013;38:67-73

32. Hou Y, Zhang S, Wang L, et al. Estrogen regulates iron homeostasis through governing hepatic hepcidin expression via an estrogen response element. Gene. 2012;511(2):398-403.

33. Yang Q, Jian J, Katz S, et al. 17 beta-Estradiol inhibits iron hormone hepcidin through an estrogen responsive element half-site. Endocrinology. 2012;153(7):3170-3178.
34. Erikson KM, Pinero DJ, Connor JR, et al. Regional brain iron, ferritin and transferrin concentrations during iron deficiency and iron repletion in developing rats. J Nutr. 1997;127(10):2030-2038.

35. Edgerton VR, Bryant SL, Gillespie CA, et al. Iron deficiency anemia and physical performance and activity of rats. J Nutr. 1972;102(3):381-399.

36. Glover J, Jacobs A. Activity pattern of iron-deficient rats. $\mathrm{Br}$ Med J. 1972;2(5814):627-628.

37. Li Y, Kim J, Buckett PD, et al. Severe postnatal iron deficiency alters emotional behavior and dopamine levels in the prefrontal cortex of young male rats. J Nutr. 2011;141(12):2133-2138.

38. Cordova FM, Aguiar AS, Peres TV, et al. In vivo manganese exposure modulates Erk, Akt and Darpp-32 in the striatum of developing rats, and impairs their motor function. PLoS One. 2012;7(3):e33057.

39. Vezer T, Kurunczi A, Naray M, et al. Behavioral effects of subchronic inorganic manganese exposure in rats. Am J Ind Med. 2007;50(11):841852 .

40. Seidler RD, Bernard JA, Burutolu TB, et al. Motor control and aging:links to age-related brain structural, functional, and biochemical effects. Neurosci Biobehav Rev. 2010;34(5):721-733.

41. Yamamoto K, Kobayashi N, Yoshitama K, et al. Isolation and purification of tyrosine hydroxylase from callus cultures of Portulaca grandiflora. Plant Cell Physiol. 2001;42(9):969-975.

42. Zhang D, Kanthasamy A, Anantharam V, et al. Effects of manganese on tyrosine hydroxylase (TH) activity and $\mathrm{TH}-$ phosphorylation in a dopaminergic neural cell line. Toxicol Appl Pharmacol. 2011;254(2):6571 .

43. Sriram K, Lin GX, Jefferson AM, et al. Dopaminergic neurotoxicity following pulmonary exposure to manganese-containing welding fumes. Arch Toxicol. 2010;84(7):521-540.

44. Unger EL, Paul T, Murray-Kolb LE, et al. Early iron deficiency alters sensorimotor development and brain monoamines in rats. J Nutr. 2007;137(1):118-124.

45. Yokel RA, Crossgrove JS. Manganese toxicokinetics at the blood-brain barrier. Res Rep Health Eff Inst. 2004;(119):7-58.

46. Fitsanakis VA, Piccola G, Marreilha dos Santos AP, et al. Putative proteins involved in manganese transport across the blood-brain barrier. Hum Exp Toxicol. 2007;26(4):295-302.

47. Fitsanakis VA, Piccola G, Aschner JL, et al. Characteristics of manganese $(\mathrm{Mn})$ transport in rat brain endothelial (RBE4) cells, an in vitro model of the blood-brain barrier. Neurotoxicology. 2006;27(1):60-70.

48. Fitsanakis VA, Piccola G, Aschner JL, et al. Manganese transport by rat brain endothelial (RBE4) cell-based transwell model in the presence of astrocyte conditioned media. J Neurosci Res. 2005;81(2):235-243.

49. Monteilh Zoller MK, Hermosura MC, Nadler MJ, et al. TRPM7 provides an ion channel mechanism for cellular entry of trace metal ions. J Gen Physiol. 2003;121(1):49-60.

50. EPA EPA. Manganese (CASRN 7439-96-5). 2010.

51. Kolasiewicz W, Kuter K, Nowak P, et al. Lesion of the cerebellar noradrenergic innervation enhances the harmaline-induced tremor in rats. Cerebellum. 2011;10(2):267-280.

52. Ikai Y, Takada M, Shinonaga Y, et al. Dopaminergic and nondopaminergic neurons in the ventral tegmental area of the rat project, respectively, to the cerebellar cortex and deep cerebellar nuclei. Neuroscience. 1992;51(3):719-728.

53. Morse AC, Beard JL, Azar MR, et al. Sex and genetics are important cofactors in assessing the impact of iron deficiency on the developing mouse brain. Nutr Neurosci. 1999;2(5):323-335. 
54. Li D. Effects of iron deficiency on iron distribution and gammaaminobutyric acid (GABA) metabolism in young rat brain tissues. Hokkaido Igaku Zasshi. 1998;73(3):215-225.
55. Finkelstein Y, Milatovic D, Aschner M. Modulation of cholinergic systems by manganese. Neurotoxicology. 2007;28(5):1003-1014. 